\title{
Dissociating the generation stage in implicit and explicit memory tests: Incidental production can differ from strategic access
}

\author{
MARY SUSAN WELDON and HERBERT L. COLSTON \\ University of Califormia, Santa Cruz, Califormia
}

\begin{abstract}
An assumption of the generate/recognize model of direct and indirect memory is that the generation stage is identical on explicit and implicit tests. Two experiments were conducted to examine the generation stage by requiring subjects to write down every word-stem solution they could generate on either an implicit test, a cued-recall test, or a generate/recognize test. In Experiment 1, the subjects studied words and anagrams; target generation was not significantly different on the three tests. However, in Experiment 2, the subjects studied the target words with a context word, and saw either the same or different context with the test stem. Now the generation stages dissociated, such that the context manipulation had no significant effect on the implicit test, but on the cued-recall test, more targets were generated with the same context words than were generated with different context words. The results argue against the claim that dissociations between implicit and explicit tests are due only to the addition of recognition processes on the explicit test, because the generation processes themselves can be dissociated.
\end{abstract}

The goal of the present research was to investigate whether information access during intentional retrieval differs from that during incidental retrieval (Jacoby, 1984). On an explicit memory test, a person deliberately tries to remember an experience. However, on an implicit test, an individual is not told that the test is related to previously studied materials; priming (facilitation) produced by the earlier experience is the index of memory. These tests are dissociable, such that many variables that have one pattern of effects on explicit tests have no effect or opposite effects on implicit tests, suggesting that different processes or subsystems underlie these different modes of retrieval (Graf \& Schacter, 1985; see reviews by Richardson-Klavehn \& Bjork, 1988; Roediger \& McDermott, 1993; Schacter, 1987).

To account for such dissociations, Jacoby and Hollingshead (1990) proposed a generate/recognize model of memory, in which intentional retrieval involves two stages (generation of candidates, plus a recognition decision), but incidental retrieval involves only the generation stage. Dissociations are said to result from the recognition stage, because the generation stages are assumed to be identical (when the tests use identical cues). To illustrate, Jacoby and Hollingshead's subjects either named five-letter words (e.g., plaza) or solved anagrams (e.g., al pzza) by rearrang-

This research was supported by a faculty research grant and a Social Sciences Divisional Research Award from the University of California, Santa Cruz. We thank Tajma Abrams for her assistance in testing subjects, and James Neely, Bradford Challis, John Gardiner, Larry Jacoby, and Henry L. Roediger III, for their many helpful comments on the manuscript. Correspondence should be addressed to M. S. Weldon, Department of Psychology, University of California, Santa Cruz, CA 95064 (e-mail: weldon@cats.ucsc.edu). ing the nonunderlined letters. Stems had either single or multiple possible solutions, but the subjects wrote only one solution for each. The subjects then received one of three tests with word stems as cues (e.g., pla--). On the implicit stem-completion test, the subjects filled in the dashes to form the first word that came to mind, which presumably involved only generation. On the generate/ recognize test, the subjects completed the same task, but then decided whether they recognized the word as previously studied. On the cued-recall test, the subjects used each word stem to help them recall a word or anagram from the study list. The latter two explicit tests were postulated to involve both the generation and recognition stages.

We consider the results for multiple-solution stems, since that type was used in our experiments. On the implicit test, intact words produced more priming than anagrams, but on the explicit tests, anagrams produced better performance than intact words. Jacoby and Hollingshead (1990) argued that implicit stem completion is a perceptual test, so intact words produced more priming than anagrams because words are perceptually more similar to the stems. However, recognition benefits from elaborative encoding, and on the explicit tests, therefore, recognition was better for the anagrams because they had been encoded more extensively. Thus, the encoding manipulation had opposite effects on the implicit and explicit tests, and this dissociation was attributed to the recognition stage on the explicit tests.

Our interest concerns the assumption that the generation processes are the same on explicit and implicit tests. As one piece of supporting evidence, Jacoby and Hollingshead (1990) compared the numbers of targets generated in the generate/recognize and implicit test conditions, and found them to be very close. However, the key piece of ev- 
idence was that for single-completion stems (e.g. cry-- for crypt), cued recall never surpassed implicit stem-completion levels. This would have to be true in a two-stage generate/ recognize model, because only items that are successfully generated can be subjected to a recognition decision. Therefore, the level of generation, as indexed by performance in the implicit stem-completion test, provides the upper limit of performance on the cued-recall test. Note that this logic cannot be applied to multiple-solution stems because on the cued-recall test, subjects can covertly generate solutions until they recognize the target, thus exceeding target production in the implicit test condition. Overall, the evidence for equivalent generation processes was quite indirect, and we sought to test this assumption more directly.

\section{EXPERIMENT 1}

Experiment 1 was essentially a replication of Jacoby and Hollingshead (1990), but with a new test method. Subjects studied intact words or anagrams (e.g., frantic or fantirc) and then received word-stem cues (e.g. fra__ an implicit stem-completion test, a generate/recognize test, or a cued-recall test. The critical feature of our test procedure was that in all test conditions, the subjects were told to write down every stem solution that came to mind (e.g., frantic, fragile, fragrant, etc.), which enabled us to examine the generation process more directly. Note that the method necessitated the use of only multiple-solution word stems.

In standard test methods (e.g., Jacoby \& Hollingshead, 1990), subjects write down only a single answer for each stem in all test conditions, so the generation process is hidden, particularly in the cued-recall test, in which subjects write only items that are both generated and recognized. Thus, it is not clear how subjects arrive at the solutions they produce, whether they generate multiple candidates, and what types of items they generate. The advantage of our method is that it reveals how items are produced during the generation stage, enabling comparisons to be made across different retrieval conditions. If the generation stages are identical, then in all test conditions (1) the encoding manipulation should have the same effect, (2) the same number of targets should be produced, and (3) the targets should be produced in the same output position.

\section{Method}

Subjects and Design. The subjects were 90 undergraduates at the University of California, Santa Cruz. All were native English speakers with normal or corrected vision. The experiment was a $3 \times 3$ mixed design, with encoding condition (read, anagram, nonstudied) manipulated within subjects and test type (implicit stem completion, explicit stem-cued recall, word-stem generate/recognize) manipulated between subjects. Thirty subjects served in each test condition.

Materials. The materials consisted of 45 six-eight-letter words of moderate frequency (10-50 per million in Kučera \& Francis, 1967), that were selected from Gibson and Watkins (1988). Word stems comprised the first three letters of each word, were unique within the group, and allowed 10 or more completions. Anagrams had the first and last letters in their correct positions. To ensure ease of solution, the first and last letters were underlined and the number of syllables in the solution was presented to the right (e.g., uphanpy 3). Words were presented similarly (e.g., unhappy 3).

For the study lists, items were randomly divided into three sets of 15 , which were counterbalanced across encoding conditions to create three lists, each studied by 15 subjects per test condition. Words and anagrams were randomly mixed, and 3 buffer items were presented at the beginning and end. The total list length was 36 items.

The test contained 3-letter stems for all targets (e.g., unh with 20 copies of each stem typed in a block.

Procedure. The subjects were tested in groups of one to five. They read the words and solved the anagrams to themselves, and were not told there would be a test. They were told that the first and last letters of all items were underlined, and that for the anagrams, these letters were in their correct positions with the number of syllables noted to the right. Subjects indicated whether they had successfully solved the anagram or read the word by circling either yes or no on a sheet of paper. Items were presented on slides in Courier font for $12 \mathrm{sec}$ each. The subjects completed two questionnaires for $8 \mathrm{~min}$ as a distractor task. The subjects were treated identically until the test phase.

Subjects performed one of three stem-completion tests. For implicit word-stem completion, they wrote as many solutions as possible. For explicit stem-cued recall, they used the word stems as cues to help them recall the words and anagrams. They wrote all solutions that came to mind until they recalled the target, then circled it and discontinued writing. For the generate/recognize test, the subjects wrote as many solutions as possible, but circled any they recognized as items presented earlier. In all test conditions, the subjects were given $30 \mathrm{sec}$ to work on each stem.

\section{Results and Discussion}

Table 1 presents the average number of solutions generated, the proportion of targets generated, and the ordinal

Table 1

Results of Experiment 1

\begin{tabular}{|c|c|c|c|c|c|c|c|c|c|}
\hline & \multicolumn{3}{|c|}{ Total Output* } & \multicolumn{3}{|c|}{ Proportion Correct $\uparrow$} & \multicolumn{3}{|c|}{ Target Position: } \\
\hline & Words & Anagrams & Nonstudied & Words & Anagrams & Nonstudied & Words & Anagrams & Nonstudied \\
\hline \multicolumn{10}{|l|}{ Targets generated $\S$} \\
\hline Implicit stem completion & 3.2 & 3.3 & 3.2 & .54 & .57 & .30 & 1.7 & 1.8 & 2.0 \\
\hline Explicit stem-cued recall & & & & .56 & .61 & .30 & 1.4 & 1.4 & 1.7 \\
\hline Generate/recognize & & & & .54 & .58 & .32 & 1.8 & 1.7 & 1.9 \\
\hline \multicolumn{10}{|l|}{$\begin{array}{l}\text { Targets generated and } \\
\text { recognized } \|\end{array}$} \\
\hline Explicit stem-cued recall & 2.2 & 2.0 & 2.6 & .38 & .50 & $.05 \#$ & 1.3 & 1.3 & $1.5 \#$ \\
\hline Generate/recognize & 3.4 & 3.5 & 3.3 & .28 & .38 & $.02 \#$ & 1.7 & 1.7 & $1.1 \#$ \\
\hline
\end{tabular}

*Total number of words produced in each condition. †Proportion of targets produced in each condition. †Position in which the target appeared among all the words produced. \$All targets produced on each test, regardless of whether they were recognized. \|Targets that were correctly recognized on the explicit tests. \#These items constitute false recognitions. 
position in which the targets were generated. The data are divided into two measures. First, target generation was obtained from all three tests (first three rows of table). For the cued-recall and generate/recognize tests, this included all targets that the subject produced, regardless of whether they were recognized, and thus represents the generation stage. The second measure (last two rows of table) consists of the proportion of targets both generated and recognized, obtained by counting only the targets that the subjects circled on the cued-recall and generate/recognize tests. The results are reported at $p<.05$.

The total output data reveal lower output in cued recall, indicating that the subjects followed instructions to stop generating when they had recalled the target.

Proportion correct: Targets generated. The data in the top three rows of Table 1 assess the generation stage in the three test conditions. The proportion-correct data suggest that the generation process was not substantially different in the implicit and explicit test conditions. Approximately equal numbers of target words and anagrams were generated on all tests. In addition, the baseline rate at which nonstudied items were generated was virtually identical on all three tests, suggesting that the subjects in the cued-recall and generate/recognize tests did not withhold responses relative to the implicit test subjects. A $3 \times 3$ analysis of variance (ANOVA) revealed a significant effect of encoding condition $\left[F(2,174)=96.51, M S_{\mathrm{e}}=4.85\right]$. There was no main effect of test $(F<1)$, and no interaction between encoding condition and test type $(F<1)$, indicating that performance was not significantly different in the three test conditions. Newman-Keuls tests revealed that on all three tests, words and anagrams produced significant priming, but were not significantly different from one another. These data are consistent with the notion that the generation stage was identical in the implicit and explicit tests.

Jacoby and Hollingshead (1990) found an advantage of read items over anagram items in their implicit test, whereas we found these conditions to be equal. One possible explanation is that our anagrams were orthographically more similar to the test stems because they retained the first letter in the correct position, whereas Jacoby and Hollingshead's anagrams did not. The first letter is a powerful cue for generating solutions, and this orthographic match may have mitigated the anagram disadvantage that Jacoby and Hollingshead observed. Further, note that some other studies also have reported equal priming from words and anagrams (e.g., Jacoby \& Hollingshead, 1990; Jacoby, Toth, \& Yonelinas, 1993). Regardless, equal performance on words and anagrams does not undermine our conclusions, because we obtained this same pattern on all three tests, and also obtained the critical dissociation when the recognition stage was added (see below).

Proportion correct: Targets generated and recognized. A $2 \times 3$ ANOVA of the proportion of targets both generated and recognized revealed a significant effect of encoding condition $\left[F(2,116)=130.62, M S_{\mathrm{e}}=4.43\right]$ and a main effect of test type $\left[F(1,58)=7.63, M S_{\mathrm{e}}=9.13\right]$, but no significant interaction $\left[F(2,116)=1.70, M S_{\mathrm{e}}=4.43\right.$, $p=.19]$. Overall performance was higher on the cuedrecall test $(M=.31)$ than on the generate/recognize test $(M=.23)$. These findings replicate Jacoby and Hollingshead (1990) in showing that on the explicit tests, when the recognition stage was added, anagrams were recalled and recognized better than words.

Target position. The three far right columns in Table 1 indicate the average ordinal position in which the targets were generated. To determine whether targets were retrieved earlier during intentional retrieval, a $3 \times 3$ ANOVA compared targets generated on the implicit test with targets generated and recognized on the explicit tests (it was assumed that these best represent items intentionally retrieved). There was no main effect of encoding condition $(F<1)$, there was a main effect of test $[F(2,87)=9.42$, $\left.M S_{\mathrm{e}}=.41\right]$, and there was a marginally significant interaction $\left[F(4,131)=2.23, M S_{\mathrm{e}}=.19, p<.10\right]$. Thus, although there appears to be a tendency to produce the words and anagrams sooner on the cued-recall test relative to the implicit test, closer inspection reveals that the nonstudied items are also produced sooner. This seems less absurd if one considers that these are false alarms, and there may be a tendency to recognize falsely items that are produced fluently (e.g., Whittlesea, 1993), which would be those produced first. To assess this possibility, we examined the target positions of nonstudied items that were generated but not recognized on the explicit tests, but found that even these were slightly lower than the nonstudied target position on the implicit tests (cued recall $=$ 1.78 , generate/recognize $=2.01$, implicit $=2.04$ ). Therefore, a fluency misattribution does not entirely account for the earlier target position of nonstudied items in the explicit tests. Also note that floor effects in this measure (1 is the lowest possible score) may preclude detection of significant effects. Finally, some subjects did not generate any targets in some conditions, rendering the measure less stable. In sum, the target-position measure does not provide strong evidence that the generation stages differed.

\section{EXPERIMENT 2}

Experiment 1 was consistent with Jacoby and Hollingshead's (1990) version of the generate/recognize model which assumes that the generation stages are identical on direct and indirect tests. However, this conclusion rests on accepting the null hypothesis, and warranted further examination. In particular, the word stems may not have been particularly useful in providing efficient intentional retrieval because (1) they are not particularly unique cues, since all words share a limited number of letters, bigrams, and trigrams; and (2) relative to words, they are not particularly meaningful, and meaningful cues can facilitate retrieval.

We hypothesized that the generation processes should differ in a situation in which meaningful contextual information could aid retrieval. Meaningful cues can be used strategically to help one retrieve information (e.g., Thomson \& Tulving, 1970). Furthermore, Weldon, Roediger, Beitel, and Johnston (1995) demonstrated that intentional 
retrieval relies on conceptual processes more than does incidental retrieval. Thus, in intentional retrieval, meaningful contextual information might be used more strategically than in incidental retrieval to access an episode efficiently and directly, an effect that would be manifest in the generation stage.

In this experiment, the subjects studied weak associates in one of two contexts (e.g., ocean-wave or sea-wave). At test, they received a two-letter stem in one of the contexts (e.g., ocean-wa___ , such that the test context was either the same as or different from the study context. They then received either an implicit or a cued-recall test.

We hypothesized that the subjects would be more likely to use contextual information when intentionally trying to retrieve an episode, so context would be more likely to affect how items were accessed and generated on the explicit test than on the implicit test. Graf and Schacter (1985) showed that with moderately related word pairs studied superficially, context changes did not hurt performance on an implicit stem-completion test. However, study-test context changes do hurt performance on explicit tests such as recognition (e.g., Light \& Carter-Sobell, 1970; Thomson, 1972) and recall (e.g., Thomson \& Tulving, 1970; Tulving $\&$ Osler, 1968). Therefore, the context manipulation should produce a dissociation between the generation stages of the implicit and explicit tests, such that context affects intentional but not incidental generation. The generate/ recognize test condition was not included in this experiment because the stem-completion and cued-recall tests provide sufficient information to test the hypothesis.

\section{Method}

Subjects and Design. The subjects were 90 undergraduates from the University of California, Santa Cruz, participating for course credit. None had participated in Experiment 1. All were native English speakers with normal or corrected vision. The experiment was a $3 \times 2$ mixed design, with study-test context (same context, different context, nonstudied) manipulated within subjects and test type (implicit stem completion, explicit stem-cued recall) manipulated between subjects. Forty-five subjects participated in each test condition.

Materials. Twenty-one word triplets were created from the Bilodeau and Howell (1965) free-association norms. The target (e.g., time) was a weak associate of the other two words (e.g. short, long), such that the probability of producing the target was .03 or less for each. Word stems were the first two letters of each target (e.g., $t i \_$_). The stems were short because the other constraints produced a small pool of short words. Each stem was unique in the set of all context and target words, and had at least 10 possible completions.

Each target was randomly assigned to one of three sets that were counterbalanced across encoding-context conditions to create three lists, each studied by 15 subjects per test condition. Items were randomly ordered in each list, with 3 buffer pairs at the beginning and end of each list. Total list length was 20 word pairs.

One of the context words was randomly selected to provide the test context. The test contained all 21 target context-stem pairs (e.g., short-ti___. Each context-stem pair was printed 12 times in a block. Nine filler items were included but not scored.

Procedure. The subjects were tested in groups of one to five. In the study phase, the subjects saw each word pair on a slide for $6 \mathrm{sec}$, and wrote the total number of vowels in both words. They were not told that they would receive a memory test. The subjects solved mazes for $5 \mathrm{~min}$ as a distractor task. They were treated identically until the test phase.

The subjects received one of two test instructions. For the implicit test, they wrote as many solutions as possible for each stem. They were told the cue word might help them think of solutions, but that they should not rely on it exclusively or it might make it difficult to think of all possible solutions. For the cued-recall test, they were told that they were receiving a memory test for the second word in each pair they had studied earlier. They would see a context word that might or might not be the one presented earlier, so they might or might not find it helpful. They wrote all the solutions they generated until they remembered the target, then circled it and stopped. Solutions did not have to be related to the context word. The subjects were given $30 \mathrm{sec}$ to work on each stem.

\section{Results and Discussion}

The data are presented in Table 2 . The results are reported at $p<.05$. The total-output measure indicates that subjects in the cued-recall condition followed instructions to stop writing when they recalled the target.

Proportion correct: Targets generated. The proportioncorrect data presented in Table 2 for the targets generated (the first two rows) indicate that the generation stages differed in the implicit and explicit tests. On the implicit test, the context manipulation had minimal effect, whereas on the explicit test, more items were generated when the study-test context was the same than when it was different. A $3 \times 2$ ANOVA revealed a main effect of context $\left[F(2,176)=23.67, M S_{\mathrm{e}}=1.63\right]$ and also a main effect of test $\left[F(1,88)=9.86, M S_{\mathrm{e}}=2.65\right]$, such that more targets were generated on the explicit cued-recall test. Most importantly, there was a significant interaction between context and test type $\left[F(2,176)=5.80, M S_{\mathrm{e}}=1.63\right]$, because

Table 2

Results of Experiment 2

\begin{tabular}{|c|c|c|c|c|c|c|c|c|c|}
\hline & \multicolumn{3}{|c|}{ Total Output* } & \multicolumn{3}{|c|}{ Proportion Correct $\dagger$} & \multicolumn{3}{|c|}{ Target Position $\ddagger$} \\
\hline & $\begin{array}{c}\text { Same } \\
\text { Context }\end{array}$ & $\begin{array}{c}\text { Different } \\
\text { Context }\end{array}$ & Nonstudied & $\begin{array}{c}\text { Same } \\
\text { Context }\end{array}$ & $\begin{array}{c}\text { Different } \\
\text { Context }\end{array}$ & Nonstudied & $\begin{array}{c}\text { Same } \\
\text { Context }\end{array}$ & $\begin{array}{c}\text { Different } \\
\text { Context }\end{array}$ & Nonstudied \\
\hline $\begin{array}{l}\text { Targets generated } \S \\
\text { Implicit stem completion } \\
\text { Explicit stem-cued recall }\end{array}$ & 5.8 & 6.0 & 6.0 & $\begin{array}{l}.48 \\
.67\end{array}$ & $\begin{array}{l}.44 \\
.51\end{array}$ & $\begin{array}{l}.38 \\
.39\end{array}$ & $\begin{array}{l}2.1 \\
1.4\end{array}$ & $\begin{array}{l}2.2 \\
1.6\end{array}$ & $\begin{array}{l}2.4 \\
1.9\end{array}$ \\
\hline $\begin{array}{l}\text { Targets generated and } \\
\text { recognized } \| \\
\text { Explicit stem-cued recall }\end{array}$ & 2.7 & 3.6 & 4.1 & .50 & .28 & $.11 \#$ & 1.2 & 1.2 & $1.8 \#$ \\
\hline
\end{tabular}

*Total number of words produced in each condition. †Proportion of targets produced in each condition. †Position in which the target appeared among all the words produced. §All targets produced on each test, regardless of whether or not they were recognized. "Targets that were correctly recognized on the explicit test. \#These items constitute false recognitions. 
the context manipulation had a larger effect on target generation during the explicit test than during the implicit test. Planned comparisons revealed that on the implicit test, performance in the same and different context conditions was not significantly different $(F<1$; replicating Graf \& Schacter, 1985), but on the explicit test, performance was significantly better in the same-context condition than in the different-context condition $[F(1,44)=$ $\left.17.22, M S_{\mathrm{e}}=25.60\right]$. Therefore, the context manipulation produced a dissociation between the generation stages on the implicit and explicit tests, indicating that generation processes during intentional retrieval differed from those during incidental retrieval. The baseline rates for generating nonstudied targets are virtually identical, arguing against the possibility that test differences are due simply to differences in subjects' criteria for generating items on the tests.

Proportion correct: Targets generated and recognized. The data in the bottom row of Table 2 indicate that the context manipulation had a significant effect on the cued-recall test, as expected. A one-way ANOVA revealed a significant context effect $\left[F(2,132)=34.15, M S_{\mathrm{e}}=2.48\right]$, and a Newman-Keuls test revealed that significantly more items were recalled in the same-context condition than in the different-context condition.

Target position. The target-position data suggest that subjects tended to produce targets earlier on the explicit test, but the data also present difficulties in interpretation similar to those in Experiment 1. Our conclusion is that the measure was not sufficiently reliable to contribute theoretically useful data in these experiments, and more work is needed to understand its potential value.

\section{GENERAL DISCUSSION}

Two experiments were conducted to examine whether items are accessed in a similar fashion during intentional and incidental retrieval The generate/recognize model (Jacoby \& Hollingshead, 1990) postulates that generation processes are the same during direct and indirect retrieval, and that dissociations between implicit and explicit tests are due to the addition of recognition processes on explicit tests. Experiment 1 replicated Jacoby and Hollingshead's encoding manipulation (words vs. anagrams), but used a new test method in which subjects were required to write down every stem solution they generated on the implicit and explicit tests. When all targets generated were assessed, regardless of whether or not they were recognized performance was nearly indistinguishable in the implicit, cued-recall, and generate/ recognize tests, supporting Jacoby and Hollingshead's thesis.

In Experiment 2, the targets were presented with a context word during the study phase, and then tested with either the same context word or a different one. Now the generation stages in the implicit and explicit tests were dissociated. The context manipulation had no significant impact on target generation on the implicit test, whereas on the explicit test, significantly more targets were generated when the study-test context was the same than when it was different. These data suggest that the generation process during intentional retrieval differs from that during incidental retrieval.

What factors might account for the different outcomes of the two experiments? We hypothesized that Experiment 2 provided meaningful context-specific information during encoding and retrieval, which could be used strategically to access targets more directly and efficiently. First, the word pairs were weakly related, which could have enabled meaningful relational processing between the context and target words during encoding. Second, the test context word was intended to increase the probability that episode-specific contextual information would play a role in retrieval, probably by limiting the search set and increasing the probability of recovering the target. The data are consistent with our interpretation because more targets were produced during the generation stage on the explicit test than on the implicit test (Table 2), and context had a bigger effect on target generation on the explicit test than it did on the implicit test. Therefore, it appears that information can in fact be accessed differently during direct and indirect retrieval, and dissociations between direct and indirect retrieval may not be due to recognition processes alone.

Given that generation processes can differ on implicit and explicit tests, it is necessary to distinguish between different types of information retrieval. In the title of our paper, we have used the terms incidental production and strategic access. Incidental production implies a more passive or automatic process, whereas strategic access implies a controlled, guided search. Our data suggest that intentional access is efficient when the test cue provides episode-specific semantic information, which can be used strategically to facilitate retrieval (see Tulving \& Thomson's 1973 encoding specificity principle). However, it is possible that providing unique information of any sort as a test cue (e.g., a unique orthographic fragment) will be sufficient to facilitate target access in intentional retrieval, and future work will help to specify the functional properties of retrieval cues that produce this effect.

In conclusion, these experiments demonstrate that retrieval (or generation processes) can differ on implicit and explicit tests, and that dissociations may not be attributable only to recognition processes. The generation processes themselves can also be dissociated, particularly when an episode-specific context can be used to guide intentional access to target events.

\section{REFERENCES}

Bilodeau, E. A., \& Howell, D. C. (1965). Free association norms by discrete and continued methods (Tech. Rep. No. 1). New Orleans: Tulane University, Department of Psychology.

Gibson, J. M., \& Watkins, M. J. (1988). A pool of 1,086 words with unique two-letter fragments. Behavior Research Methods, Instruments, \& Computers, 20, 390-397.

GRAF, P., \& SCHACTER, D. L. (1985). Implicit and explicit memory for new associations in normal and amnesic subjects. Journal of Experimental Psychology: Learning, Memory, \& Cognition, 11, 501-518.

JACOBY, L. L. (1984). Incidental versus intentional retrieval: Remembering and awareness as separate issues. In L. S. Squire \& N. Butters (Eds.), Neuropsychology of memory (pp. 145-156). New York: Guilford.

JACOBY, L. L., \& HolLINGSHEAD, A. (1990). Toward a generate/recognize model of performance on direct and indirect tests of memory. Journal of Memory \& Language, 29, 433-454.

JACOBY, L. L., ToTH, J. P., \& Yonelinas, A. P. (1993). Separating conscious and unconscious influences of memory: Measuring recollection. Journal of Experimental Psychology: General, 122, 139-154.

Kư̌ERA, H., \& FrANCIS, W. N. (1967). Computational analysis of presentday American English. Providence, RI: Brown University Press.

Light, L. L., \& CARTER-SoBeLL, L. (1970). Effects of changed semantic context on recognition memory. Journal of Verbal Learning \& Verbal Behavior, 9, 1-11.

RiCHARDSON-KLAVEHN, A., \& BJORK, R. A. (1988). Measures of memory. Annual Review of Psychology, 39, 475-543.

RoEDiger, H. L., III, \& MCDERMOTT, K. B. (1993). Implicit memory in normal human subjects. In H. Spinnler \& F. Boller (Eds.), Handbook of neuropsychology (Vol. 8, pp. 63-131). Amsterdam: Elsevier.

SCHACTER, D. L. (1987). Implicit memory: History and current status. 
Journal of Experimental Psychology: Learning, Memory, \& Cognition, 13, 501-518.

Thomson, D. M. (1972). Context effects in recognition memory. Journal of Verbal Learning \& Verbal Behavior, 11, 497-511.

Thomson, D. M., \& Tulving, E. (1970). Associative encoding and retrieval: Weak and strong cues. Journal of Experimental Psychology, 86, 255-262.

TUlving, E., \& Osler, S. (1968). Effectiveness of retrieval cues in memory for words. Journal of Experimental Psychology, 77, 593-601.

Tulving, E., \& THOMSON, D. M. (1973). Encoding specificity and retrieval processes in episodic memory. Psychological Review, 80, 352-373.
Weldon, M. S., Roediger, H. L., III, Beitel, D., \& Johnston, T. R. (1995). Perceptual and conceptual processes in implicit and explicit tests with picture fragment and word fragment cues. Journal of Memory \& Language, 34, 268-285.

WHITTLESEA, B. W. A. (1993). Illusions of familiarity. Journal of Experimental Psychology: Learning, Memory, \& Cognition, 19, 1235-1253.

(Manuscript received October 21, 1994; revision accepted for publication February 16, 1995.)

\section{6th Annual Meeting of the Psychonomic Society Los Angeles, California November 10, 11, and 12, 1995}

The 36th Annual Meeting of the Psychonomic Society will be held in Los Angeles, California, November 10,11, and 12,1995, at the Westin Bonaventure Hotel. The meetings will begin Friday morning and continue until Sunday at noon.

Programs and hotel reservation cards will be mailed to members and associates in September. Additional programs will be available at the meeting registration desk for $\$ 7.00$.

For further information, please contact the Secretary-Treasurer of the Society: Randi C. Martin, Department of Psychology, Rice University, P.O. Box 1892, Houston, TX 77251-1892 (phone: 713527-8101, ext. 3417; e-mail: rmartin@rice.edu). 